\title{
Title: Modeling Complex Phenomena: Multiple Length and Time Scales in Extended Dynamical Systems
}

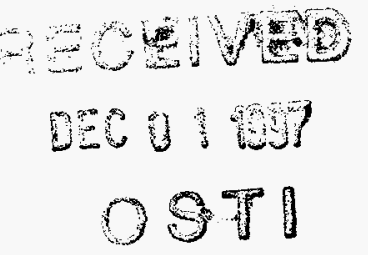

Author(s): P. Lomdahl, A. Bishop, S. D. Cai, N. G. Jensen, T-11

B. Holian, T-12; S. Zhou, X-NH

M. Samulensen, Technical University of Denmark

D. Beazley, University of Utah

D. Dominguez, Centro Atomico Bariloche, Argentina

A. Sanchez, University of Madrid

G. Wysin, Kansas State University

Submitted to:

DOE Office of Scientific and Technical Information (OSTI)

\section{Los Alamos}

DSTRHBUTON OF THIS DOCWEAT IS UNMUMED

NATIONAL LABORATORY

Los Alamos National Laboratory, an affirmative action/equal oppontunity employer, is operated by the University of Calitomia for the U.S. Department of Energy under contract W-7405-ENG-36. By acceptance of this article, the publisher recognizes that the U.S. Government retains a nonexclusive, royaltytree license to publish or reproduce the published form of this contribution, or to allow others to do so, for U.S. Government purposes. Los Alamos National Laboratory requests that the publisher identify this article as work performed under the auspices of the U.S. Department of Energy. Los Alamos National Laboratory strongly supports academic freedom and a researcher's right to publish; as an institution, however, the Laboratory does not endorse the viewpoint of a publication or guarante its technical correctness. 


\section{DISCLAIMER}

This report was prepared as an account of work sponsored by an agency of the United States Government. Neither the United States Government nor any agency thereof, nor any of their employees, makes any warranty, express or implied, or assumes any legal liability or responsibility for the accuracy, completeness, or usefulness of any information, apparatus, product, or process disclosed, or represents that its use would not infringe privately owned rights. Reference herein to any specific commercial product, process, or service by trade name, trademark, manufacturer, or otherwise does not necessarily constitute or imply its endorsement, recommendation, or favoring by the United States Government or any agency thereof. The views and opinions of authors expressed herein do not necessarily state or reflect those of the United States Government or any agency thereof. 


\section{DISCLAMIER}

Portions of this docoment mily be Illegible in electronic image products. Images are produced from the best arailable original docoment. 


\title{
Modeling Complex Phenomena: Multiple Length and Time Scales in Extended Dynamical Systems
}

\author{
Peter Lomdahl, * Alan Bishop, Niels Gronbech Jensen, \\ S. David Cai, Brad Holian, and Shujia Zhou \\ Los Alamos National Laboratory \\ Mogens Samulensen \\ Technical University of Denmark \\ David Beazley \\ University Utah \\ Daniel Dominquez \\ Centro Atomico Bariloche, Argentina \\ Angel Sanchez \\ University of Madrid, Spain \\ Gary Wysin \\ Kansas State University
}

\begin{abstract}
This is the final report of a three-year, Laboratory Directed Research and Development (LDRD) project at Los Alamos National Laboratory (LANL). Using nonlinear techniques and large-scale simulations, we have systematically studied meso-scale pattern formation and dynamics in nonlinear, nonequilibrium systems exhibiting topological excitations (dislocations, vortices, vortex lines, domain walls); dislocation generation from crack fronts in ductile materials; the smoothing of rough surfaces in solid-on-solid models; ordering and melting of moving flux lattices in threedimensional Josephson junction arrays with external magnetic field, current, and disorder; filamentary and plastic vortex flow in disordered thin films superconductors; magnetic vortices in Heisenberg spin layers; and hierarchical twinning and tweed texture in elastic models.
\end{abstract}

\section{Background and Research Objectives}

We have accumulated over the last decade a varied expertise in modern methods to handle nonequilibrium and nonlinear processes in condensed matter and materials science. In particular we have acted to translate the rapid growth of techniques in nonlinear science [e.g. at the Center for Nonlinear Science (CNLS) at Los Alamos] and in scientific computing [through the Advanced Computing Laboratory ( $\mathrm{ACL}$ ) at Los Alamos] to applications in condensed matter and materials science (e.g., microstructural process

\footnotetext{
*Principal Investigator: pxl@lanl.gov
} 
models). It is now clear that several fundamental lessons have emerged as major strategic research directions, both in the context of nonequilibrium and nonlinear science generally and in terms of specific materials science issues. In particular, nonlinear science techniques (e.g., nonlinear mode reduction) and large-scale parallel simulations can qualitatively advance our understanding of mesoscopic processes determining structure and dynamics in complex materials. We believe that this is a key ingredient in establishing the crucial bridge between microscopic and macroscopic levels of description to understand materials growth and morphology properties. The mesoscale remains a very difficult regime for experimental structural and dynamical probes, so that simulation, modeling, and measurement must work together synergistically. The major lessons are:

- The formation and dynamics of "collective" or "coherent" space-time structures, built from single particle (e.g., atomistic) dynamics, is now appreciated to be the central issue in extended dynamical systems. Coherent structures include dislocations, vortices, domain walls, twin boundaries, and clusters. These basic concepts are not new in materials science, where local structures such as dislocations, vertices, grain, and twin boundaries are known to control the macroscopic strength and response properties of materials. However, constructive nonlinear and nonequilibrium techniques to understand the microscopic origin of these textures and their collective dynamics have advanced substantially. In particular the collective structures provide a mesoscopic level of description, and their self-organizing interactions are responsible for complex patterns and dynamics.

- The above developments have clearly revealed the underlying role of competing length and frequency scales as a principle mechanism determining patterns in space and/or time. The length scales result from, e.g., the underlying lattice and the intrinsic particle-particle interactions. Competitions of length scales or symmetries typically lead to patterns ("textures") in space, which can be constant, periodic, quasiperiodic, or even totally irregular ("structural chaos"). Familiar examples include incommensurate structures observed in, e.g., surface epitaxial growth, but these are now extended to include complex textures in martensitic or elastic materials, sintering, recrystallization, etc.

- The intimate relationship between space and time noted above has only recently begun to be appreciated. Ground-state spatial structures lead directly to the many time scales observed as a macroscopic system relaxes from a nonequilibrium configuration, or as it responds to external forces. We have focused on a number of model problems drawn from condensed matter (e.g., magnetic materials, Josephson transmission lines) and 
materials science (e.g., dislocation emission from crack tips, twinned elastic materials, charge-density-wave compounds), which demonstrate this coupling of space and time.

- The importance of "noise" and "disorder" has long been appreciated in condensed matter and materials science. These effects can lead, for example, to localized modes, Anderson localization, broadening of phase transitions, hysteresis, pinning, workhardening, etc. However, the research community has only recently understood enough about these effects and those driven by strong nonlinearity to face the reality that these two features must be incorporated simultaneously to properly understand mesoscopic textures.

- Computational capabilities have now reached a stage where they share a synergistic relationship with modeling and experiment, which is qualitatively affecting our understanding of processes in materials. However, $a b$ initio solution of macroscopic properties is still far beyond computational reality. We have therefore developed codes that serve to motivate and benchmark reduced models designed to capture the essential mesoscale phenomena; the strategy is that simulations can then treat these reduced models on an even larger scale; and so on, iteratively, towards macroscopic scales. We have specifically used codes devoted to Monte Carlo (MC), Molecular Dynamics (MD), Langevin MD, solving nonlinear partial differential equations, etc.; adaptation of codes to parallel processing capabilities at the ACL (the TMC CM-5 and Cray T3D); and coupling of massively parallel computing with innovative real-time graphical visualization.

\section{Importance to LANL's Science and Technology Base and National R\&D Needs}

A central focus of the Department of Energy (DOE) and the Department of Defense (DoD) is in the area of materials synthesis and processing, an important aspect of which is to develop a capability to microscopically control synthesis and processing of materials with qualified mesoscale textures and correlated macroscopic properties.

Work on the structure and dynamics of collective structures complements many programs at the Laboratory. The work on layered magnets and structural phase transitions, for instance, provides fundamental input for inelastic neutron scattering studies at the Los Alamos Neutron Science Center (LANSCE). It is the science underlying magnetic storage technology. Again, the modeling of surface growth is directly related to spiral and whisker growth mechanisms, as studied in the Los Alamos Materials Science and Technology (MST) Division and recently observed by scanning-tunneling electron microscopy 
(STEM), as a growth mechanism for thin films of high- $\mathrm{T}_{c}$ superconductors. It is likewise a key science for semiconductor technology. By establishing mesoscopic texture links between microscopic and macroscopic properties of a material, we can help provide guidance to many programs to better design materials with reproducible properties and performance. Fracture and crack propagation are central to many such programs at the Laboratory, where an emphasis on microstructural input to process models for materials is rapidly growing.

This project was directly connected to the Laboratory core competency in theory, modeling and high-performance computing; it deliberately integrated all of these three elements in a strategy for addressing complex phenomena arising in condensed matter and materials science. Our primary focus was to develop a mathematical and computational physics technology base for handling compelling new issues in nonlinear and nonequilibrium dynamical systems theory. However, we were careful to take strong guidance from, and transfer results to, specific applications being studied experimentally at Los Alamos and elsewhere.

\section{Scientific Approach and Accomplishments}

We focused our work on four distinct areas:

- Dynamics of fracture and crack propagation studied by multimillion particle MD.

- Vortex and domain wall textures, their interactions and dynamics in ordered and disordered in two- and three-dimensional (2-D and 3-D) arrays of Josephson junctions.

- Vortex and domain wall flux patterns, their interactions and dynamics in ordered and disordered 2-D magnets and multilayers.

- Surface growth modeling and simulation, including coupling to surface chemical reactions.

Details are given below for each case, but all topics were studied with some common objectives and techniques.

Dynamics of Crack Propagation. We have studied crack propagation in both 2-D and 3-D geometries, interphases at finite temperature, and atomistic defects (dislocations) with our CM-5 MD and Langevin MD codes. We have characterized the brittle to ductile fracture transition by tuning the interatomic potentials in the MD simulations (including realistic silicon potentials). A significant new tool in understanding 
the fundamental behavior of materials that break under load is large-scale MD simulation, carried out on a massively parallel computer. The origin of blunting of a crack in a ductile metal begins at the atomic scale of the crack tip. Rather than advancing by breaking bonds between atoms, the crack can emit a dislocation (a mobile mismatch in the crystal lattice) into the bulk of the material, leaving behind a step in the crack surface. Again, using large scale simulations we have been able to characterize macroscopic friction coefficients between two surfaces modeled by atomistic (MD) interactions, and we have done preliminary work simulating ion implantation in bulk silicon. The results show that only very-large-scale simulations will capture the spatial complexity of amorphization and ion channeling of realistic implant energies.

Josephson Junction Arrays. Studies of macroscopic responses and their description in terms of collective flux structures has reached a stage of intensive experimental and theoretical activity in 2-D and 3-D arrays of superconducting Josephson junction arrays (JJA). These systems are of interest in their own right, in particular in connection with fast switching networks for supercomputers, but also as a controlled laboratory to study defect creation and macroscopic dynamics. Further, the JJA is a convenient phenomenological model for studying the dynamics of layered high-temperature superconductors. The JJA in external magnetic fields exhibit domain-wall-like and vortexlike coherent structures. We have developed several different JJA Langevin stochastic dynamics codes simulating both 2-D and 3-D dynamics of overdamped and underdamped lattices of different geometries on single and multiprocessor workstations. We have simulated the effects of disorder on driven 2-D (1024 x 1024) lattices, and vortexloop/vortex-loop interactions in 3-D (100 x $100 \times 100)$ arrays.

We have related the 2-D topological transition (Kosterlitz-Thouless) temperature to vortex loop nucleation in 3-D (anisotropic) systems. We have applied our Langevin MD approach for very large arrays to follow nonequilibrium dynamics of driven systems at finite temperature. Through these studies we have given the first theoretical understanding of the experimentally observed transformer effect in current-driven, layered hightemperature superconductors at zero magnetic field. In this case, we measure (as is done in the experiments) the voltage over the bottom plane of our array, induced by a current through the top plane. We have further identified the phase-transitions in the currenttemperature-anisotropy parameter space. In 2-D, we have studied the response functions of a driven disordered lattice in large magnetic fields, and we have identified several macroscopic current-voltage signatures for different phases of microscopic flux dynamics. We are thus able to suggest experimental measurements of, e.g., noise properties indicating, for example, plastic flow of vortices. All these results are crucially dependent 
on the ongoing development of advanced visualization capabilities for the microscopic structures. These tools allow us to follow the microscopic behavior as it evolves during a dynamical simulation of nonequilibrium flow and relaxation.

Two-Dimensional Magnets. Mesoscopic structures in layered magnets are typically domain-wall or vortex configurations. We have succeeded in implementing an analytical collective-variable ansatz for a system of $\mathrm{N}$ extended-field nonlinear excitations in 2-D magnetic systems governed by Landau-Lifshitz equations of motion. This allowed us to rigorously derive a (nonlinear) reduced model that accurately describes mesoscopic order and dynamics emerging from many coupled spins. In contrast to earlier ad hoc approaches, in which interactions between collective excitations have been treated only through an effective external forcing term, we have explicitly considered dependence of the microscopic spin field on all the coordinates and velocities of the collective excitations. We have applied this approach to vortices in 2-D Heisenberg ferromagnets with weak easyplane anisotropy. For vortex pairs, we find either rotational or translational motion, but with an additional cyclotron-like oscillation. Due to the interactions between the two vortices, we obtain two different eigenvalues of the mass- and gyro-tensors, with values that depend on the distance between the vortices, their vorticities, and the direction of their out-of-plane spin fields. This collective variable approach is in good agreement with our direct numerical simulations of spin structure and dynamics for large systems.

Dynamics of Surface Growth. We have used our large system (up to $1024 \mathrm{x}$ 1024 lattice points) Langevin MD and advanced visualization capability to study the structure and dynamics associated with surfaces driven by temperature and surface-vapor chemical potential difference. We have investigated the equilibrium Kosterlitz-Thouless roughening transition and shown that this splits into two crossovers when external forces (chemical potential) are applied. We have characterized the different regimes in terms of roughness, growth rates, and height-height correlation functions. Our results are consistent with available experiments, and we have suggested new experimental work based on our new insight. 


\section{Publications}

1. Pellegrini, M., Gronbech-Jensen, N., Doniach, S., "Potentials of mean force for biomolecular simulations: Theory and test on alanine dipeptide," J. Chem. Phys. 104, 8639 (1996).

2. Dominguez, D., Gronbech-Jensen, N., Bishop. A., "Dynamics of current driven disordered Josephson junction arrays," Macroscopic Quantum Phenomena and Coherence in Supercomputing Networks, eds. Giovannella, C. and Tinkham, M. (World Scientific, p. 278 (1995).

3. Dominguez, D., Bishop, A., Gronbech-Jensen, N., "Coherence and complexity in condensed matter: Josephson junction arrays," Int. J. Bif. Chaos, In Press (1996) LA-UR-96-3409.

4. Gronbech-Jensen, N., Dominguez, D., Bishop, A., "Simulations of current driven three dimensional Josephson junction arrays," Physica B 222, 396 (1996).

5. Zhou, S., Beazley, D., Lomdahl, P., Holian, B., "Large-Scale Molecular Dynamics Simulations of Three-Dimensional Fracture," Los Alamos Natinal Laboratory report LA-UR-96-1576) (1996).

6. Holian, B., Lomdahl, P., Zhou, S., "Fracture Simulations via Large-Scale Nonequilibrium Molecular...", Los Alamos Natinal Laboratory report LA-UR-962484 (1996).

7. Zhou, S., Beazley, D., Lomdahl, P., Voter, A., Holian, B., "Dislocation Emission from a Three-Dimensional Crack Los Alamos Natinal Laboratory report LA-UR-962487 (1996).

8. Zhou, S., Lomdahl, P., Thomson, R., Holian, B., "Dynamic Crack Processes via Molecular Dynamics," Phys. Rev. Lett. 76, 2318 (1996).

9. Cirillo, Bishop, A. Jensen, N., Lomdahl, P., "High-frequency pumping of Josephson Soliton Oscillators," Phys. Rev. B 52, 506 (1995).

10. Dominguez, D., Jensen, N., Bishop, A., Shenoy, S., "Transformer configuration in 3-dimensional Josephson Lattices...," Phys. Rev. Lett. 75, 717 (1995).

11. Sanchez, A., Bishop, A., Jensen, N., "Smoothing of Rough Surfaces," Phys. Rev. B 52, 5433 (1995).

12. Dominguez, D., Jensen, N., Bishop, A., "Resistive hysteresis and nonlinear J-V characteristics...," Phys. Rev. Lett. 75, 4670 (1995).

13. Jensen, N., Bishop, A., Dominguez, D., "Metastable filamentary vortex flow in thin film superconductors," Phys. Rev. Lett. 76, 2985 (1996).

14. Saxena, A., Shenoy, S., Bishop, A., "Hierarchical twinning: martensites as patternforming systems," Physica D (in press) (1996).

15. Mertens, F., Schnitzer, H., Bishop, A., "Hierarchy of equations of motion for nonlinear coherent excitations...," Physica D (in press) (1996). 
16. Dominguez, D., Jensen, N., Bishop, A., "First-order melting of a moving vortex lattice: effects of disorder," Phys. Rev. Lett. 78, 2644 (1997).

17. Gronbech-Jensen, N., Cirillo, M., "Phase-locking of Fiske modes in sine-Gordon systems," Phys. Rev. B 50, 12851 (1994).

18. Gronbech-Jensen, N., Samuelsen, M., "Long range magnetic interaction between Josephson junctions," Phys. Rev. Lett. 74, 170 (1995).

19. Gronbech-Jensen, N., Lomdahl, P., Cirillo, M., "Uniform coupling of microwaves to nonlinear resonant modes in Josephson junctions," Phys. Rev. B 51, 11690 (1995).

20. Salerno, M., Gronbech-Jensen, N., Samuelsen, M., "Relaxation towards phaselocked dynamics in long Josephson junctions," Phys. Rev. B 51, 15613 (1995).

21. Sanchez, A., Cai, D., Gronbech-Jensen, N., Bishop, A., Wang Z., "Roughening transitions of driven surface growth," Phys. Rev. B 51, 14664 (1995). 